Supporting Information for

\title{
Boosting Electrochemical Performance of Lithium-Rich Manganese-Based Cathode Materials Through Dual-Modification Strategy with Defect Designing and Interface Engineering
}

\author{
Zhi Li ${ }^{\mathrm{a}}$, Shuang $\mathrm{Cao}^{\mathrm{a}}$, Xin Xie ${ }^{\mathrm{a}}$, Chao $\mathrm{Wu}^{\mathrm{a}}$, Heng $\mathrm{Li}^{\mathrm{a}}$, Baobao Chang \\ Gairong Chen ${ }^{\mathrm{c}}$, Xiaowei Guo ${ }^{\mathrm{c}}$, Xiaoyan Zhang*a, Xianyou Wang*a
}

( ${ }^{a}$ National Base for International Science \& Technology Cooperation, National Local Joint Engineering Laboratory for Key Materials of New Energy Storage Battery, Hunan Province Key Laboratory of Electrochemical Energy Storage \& Conversion, School of Chemistry, Xiangtan University, Xiangtan 411105, China

${ }^{\mathrm{b}}$ Key laboratory of Materials Processing and Mold of Ministry of Education, Zhengzhou University, Henan 450001, China

c School of Chemistry \& Materials Engineering, Xinxiang University, Henan 453003, China)

*Corresponding author: Xianyou Wang, E-mail address: wxianyou@yahoo.com; Xiaoyan Zhang: kld527@,126.com. 


\section{S1. SUPPLEMENTARY FIGURES}
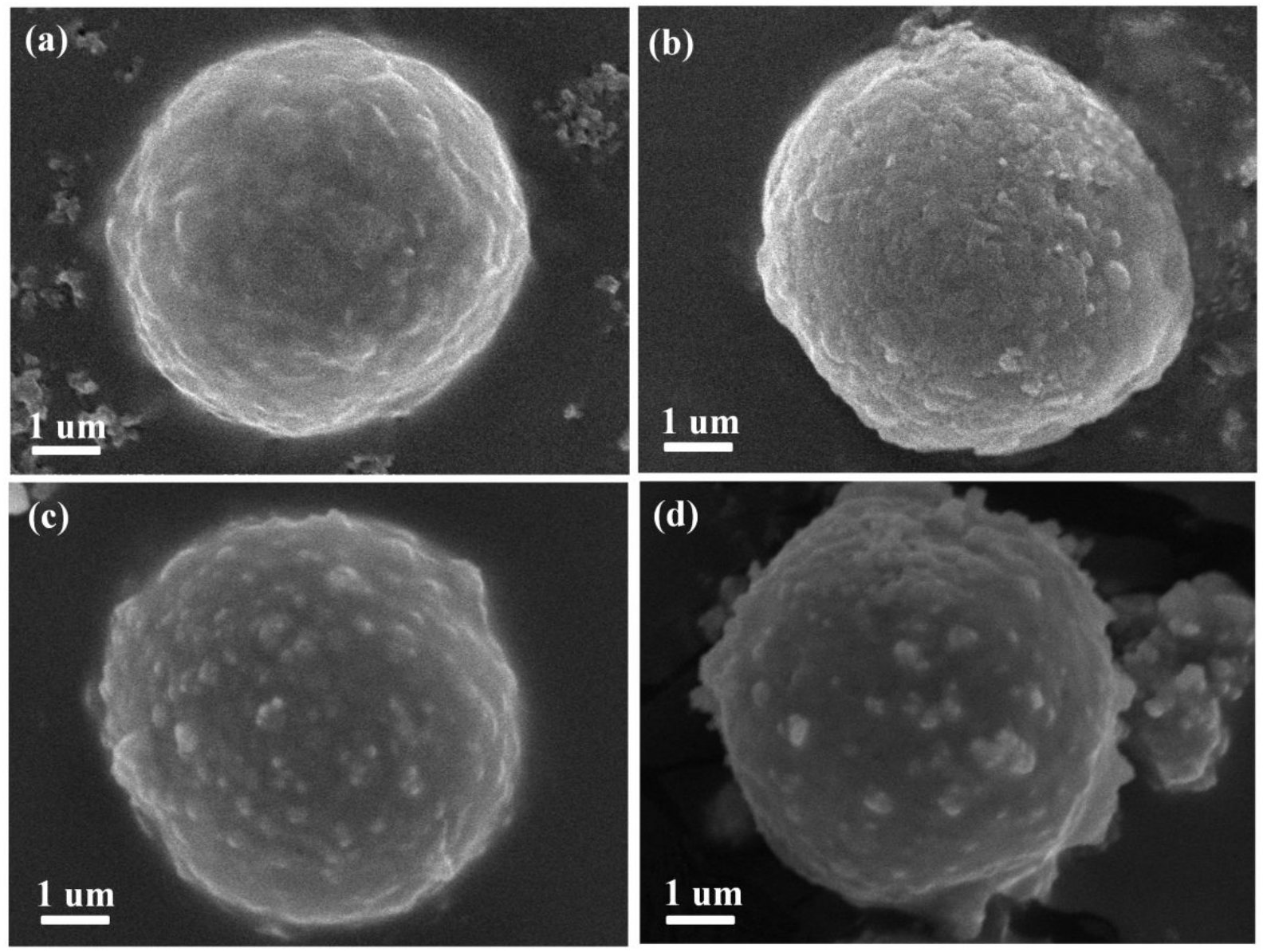

Figure S1. SEM images of the (a) carbonate precursor, (b) LMNC, (c) CN-LMNC and (d) PCN-LMNC.

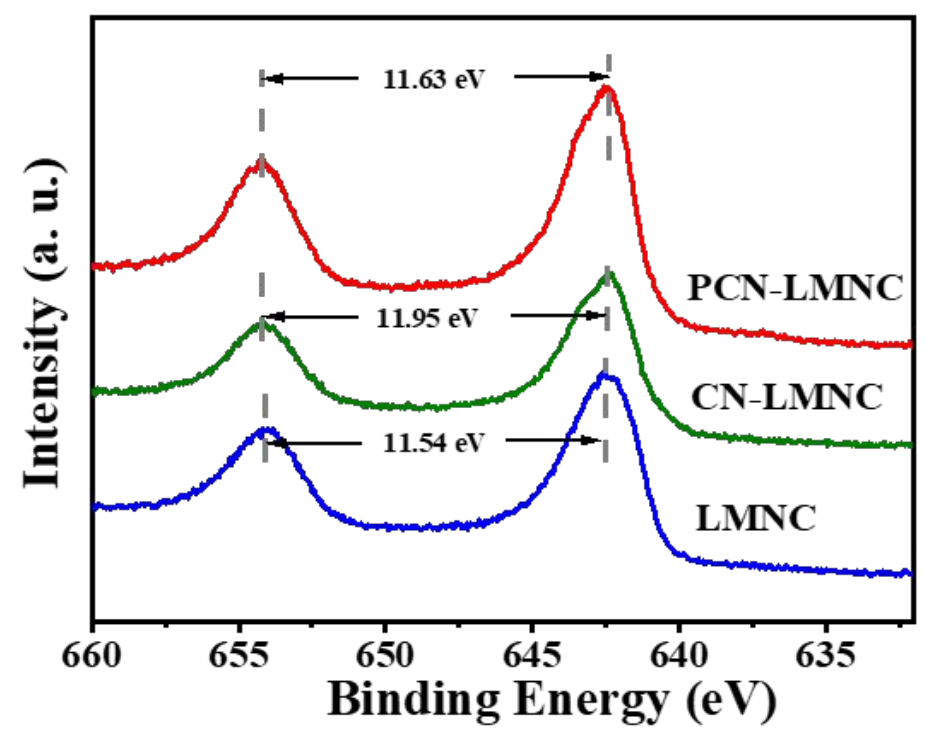

Figure S2. The high-resolutions of Mn XPS spectra. 


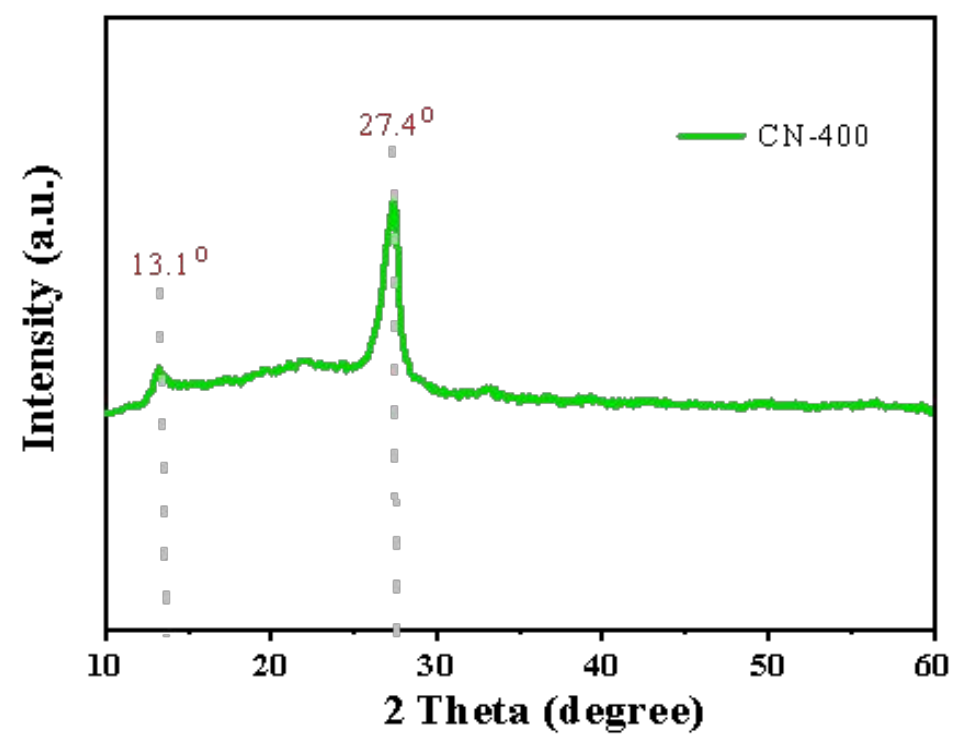

Figure S3. XRD pattern of pure carbon nitride.

(a)

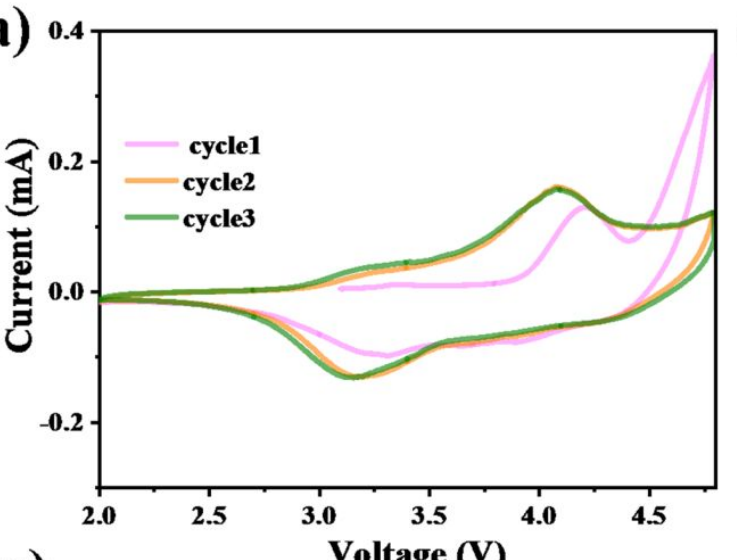

(c)

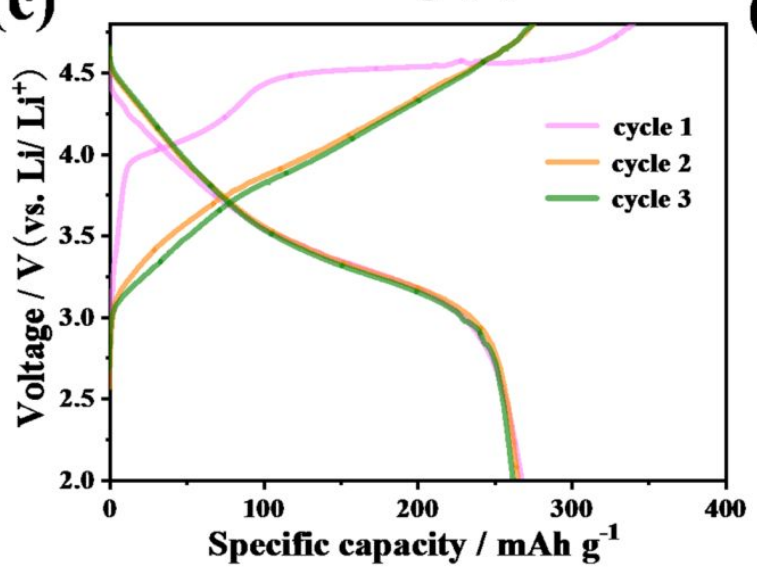

(b)

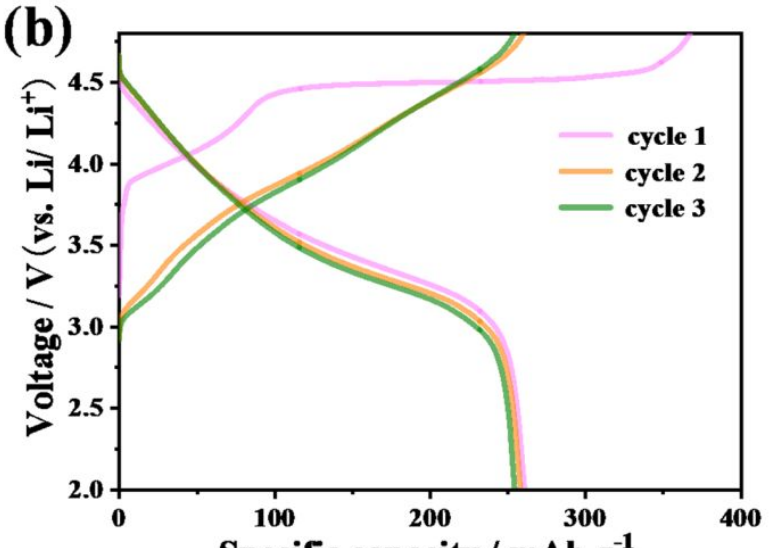

(d)

Specific capacity / mAh $\mathrm{g}^{-1}$

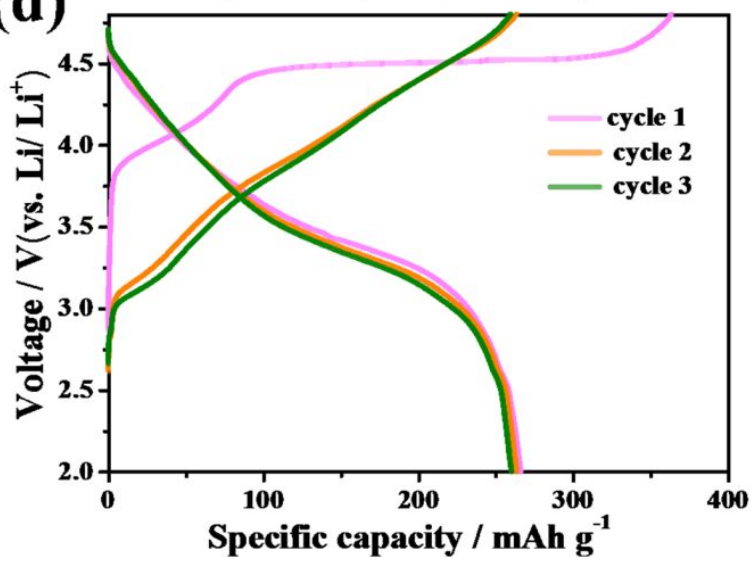

Figure S4. (a) Cyclic voltammograms (CV) of CN-LMNC. The charge-discharge curves of (b) LMNC, (c) CN-LMNC and (d) PCN-LMNC at $0.1 \mathrm{C}\left(1 \mathrm{C}=200 \mathrm{mAh} \mathrm{g}^{-1}\right)$. 

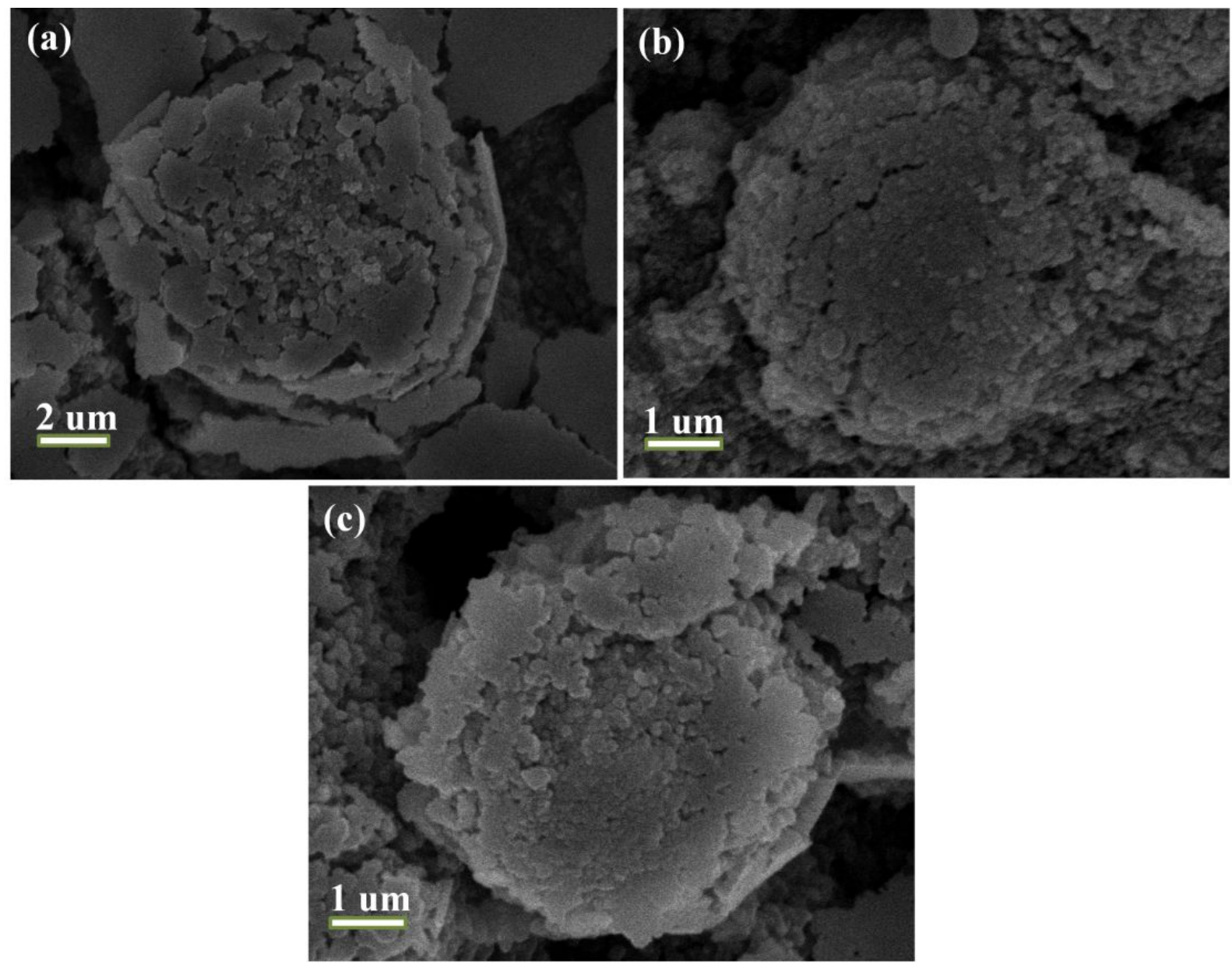

Figure S5. SEM images of (a) LMNC, (b) CN-LMNC and (c) PCN-LMNC positive electrodes after long-term cycling.
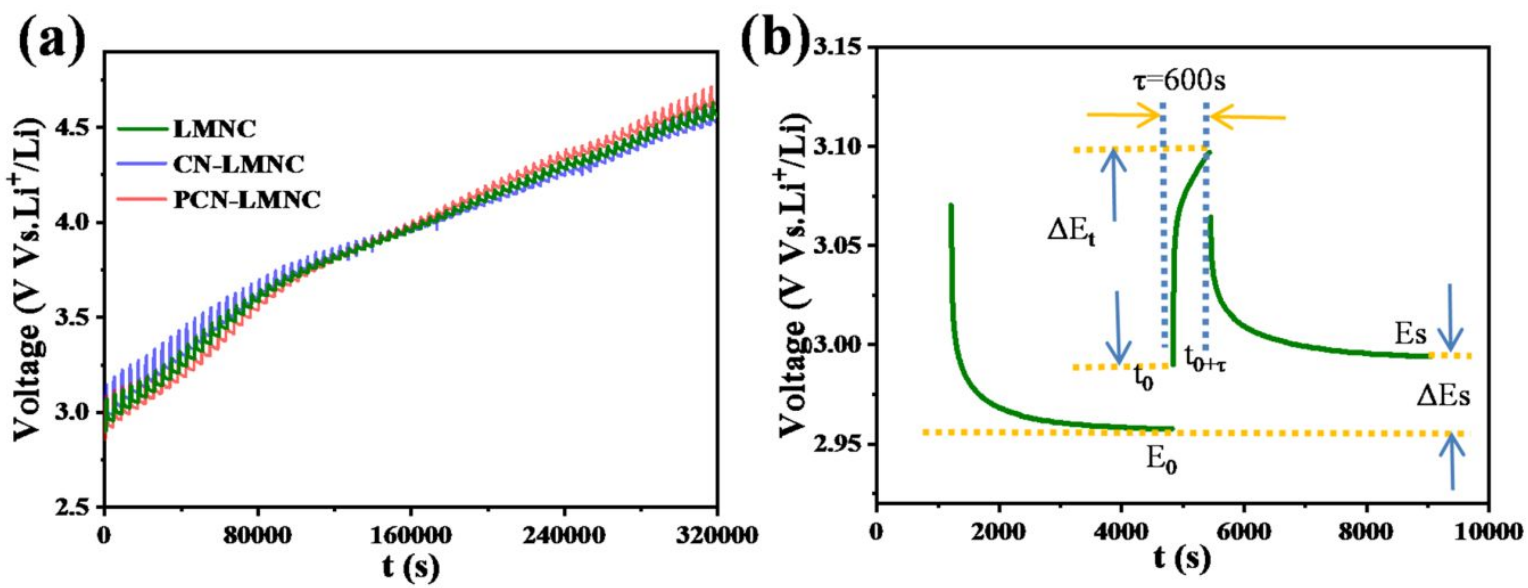

Figure S6. (a) GITT curves of LMNC, CN-LMNC and PCN-LMNC. (b) The selected t vs. V diagram of onefold GITT titration. 


\section{S2. SUPPLEMENTARY TABLES}

Table S1. The concentrations of the metal elements on the as-prepared samples are determined by Atomic Absorption Spectroscopy (AAS).

\begin{tabular}{ccccc}
\hline \multirow{2}{*}{ samples } & \multicolumn{4}{c}{ Normalized element content (use $\mathrm{Li}=1.20$ for all samples) } \\
\cline { 2 - 4 } & $\mathrm{Li}$ & $\mathrm{Mn}$ & $\mathrm{Ni}$ & $\mathrm{Co}$ \\
LMNC & 1.20 & 0.543 & 0.129 & 0.131 \\
CN-LMNC & 1.20 & 0.541 & 0.128 & 0.130 \\
PCN-LMNC & 1.20 & 0.542 & 0.127 & 0.129 \\
\hline
\end{tabular}

Table S2. Brief refine crystal parameters of LMNC and PCN-LMNC samples.

\begin{tabular}{|c|c|c|c|}
\hline \multicolumn{2}{|c|}{ parameters } & \multirow{2}{*}{$\begin{array}{c}\text { LMNC } \\
0.2900\end{array}$} & \multirow{2}{*}{$\begin{array}{c}\text { PCN-LMNC } \\
0.2902\end{array}$} \\
\hline & $a(\mathrm{~nm})$ & & \\
\hline \multirow[t]{3}{*}{$R \overline{3} m$} & $b(\mathrm{~nm})$ & 0.2900 & 0.2902 \\
\hline & $c(\mathrm{~nm})$ & 1.4478 & 1.4504 \\
\hline & $a(\mathrm{~nm})$ & 0.5007 & 0.5004 \\
\hline \multirow[t]{2}{*}{$C 2 / m$} & $b(\mathrm{~nm})$ & 0.8689 & 0.8675 \\
\hline & $c(\mathrm{~nm})$ & 0.5096 & 0.5092 \\
\hline \multirow{2}{*}{$\mathrm{Wt} \%$} & $R \overline{3} m$ & $50.52 \%$ & $51.50 \%$ \\
\hline & $C 2 / m$ & $49.48 \%$ & $48.50 \%$ \\
\hline \multirow[t]{2}{*}{ O occupancy } & & 0.9838 & 0.9362 \\
\hline & Rwp & $5.89 \%$ & $5.80 \%$ \\
\hline \multirow[t]{2}{*}{ Rietveld factor } & $\mathrm{Rp}$ & $4.64 \%$ & $4.59 \%$ \\
\hline & $X^{2}$ & 1.54 & 1.61 \\
\hline
\end{tabular}


Table S3. Electrochemical performance of LMNC, CN-LMNC and PCN-LMNC samples.

\begin{tabular}{|c|c|c|c|}
\hline Samples & $\begin{array}{c}\text { Initial discharge } \\
\text { capacity and } \\
\text { Coulombic efficiency } \\
\text { at } 0.1 \mathrm{C}\end{array}$ & $\begin{array}{l}\text { Initial discharge } \\
\text { capacity at } 1 \mathrm{C} \text { and } \\
\text { capacity retention } \\
\text { after } 150 \text { cycles }\end{array}$ & $\begin{array}{c}\text { discharge capacity } \\
\text { at } \\
5 \mathrm{C} \text { and } 10 \mathrm{C}\end{array}$ \\
\hline \multirow[t]{2}{*}{ LMNC } & $261.5 \mathrm{mAh} \mathrm{g}^{-1}$ & $228.7 \mathrm{mAh} \mathrm{g}^{-1}$ & 77.6 and 25.1 \\
\hline & $71.0 \%$ & $68.5 \%$ & $\mathrm{mAh} \mathrm{g}{ }^{-1}$ \\
\hline \multirow[t]{2}{*}{ CN-LMNC } & $268.2 \mathrm{mAh} \mathrm{g}^{-1}$ & $238.9 \mathrm{mAh} \mathrm{g}^{-1}$ & 93.5 and 31.4 \\
\hline & $78.7 \%$ & $87.9 \%$ & $\mathrm{mAh} \mathrm{g}^{-1}$ \\
\hline \multirow[t]{2}{*}{ PCN-LMNC } & $267.5 \mathrm{mAh} \mathrm{g}^{-1}$ & $230.3 \mathrm{mAh} \mathrm{g}^{-1}$ & 174.6 and 137.8 \\
\hline & $75.5 \%$ & $92.4 \%$ & $\mathrm{mAh} \mathrm{g}^{-1}$ \\
\hline
\end{tabular}

Table S4. The properties comparisons between this work with previous reports.

\begin{tabular}{cccccc}
\hline Samples & C-rate & $\begin{array}{c}\text { Cycle } \\
\text { number }\end{array}$ & $\begin{array}{c}\text { Remaining } \\
\text { Capacity } \\
\left(\mathrm{mAhg}^{-1}\right)\end{array}$ & $\begin{array}{c}\text { Rate Capacity } \\
\left(\mathrm{mAhg}^{-1}\right)\end{array}$ & Ref. \\
\hline $\mathrm{Li}_{1.2} \mathrm{Mn}_{0.534} \mathrm{Ni}_{0.133} \mathrm{Co}_{0.133} \mathrm{O}_{2}$ & $1 \mathrm{C}$ & 100 & 185 & 160 at $2 \mathrm{C}$ & {$[\mathrm{S} 1]$} \\
$\mathrm{Li}_{1.2} \mathrm{Mn}_{0.54} \mathrm{Ni}_{0.16} \mathrm{Co}_{0.1} \mathrm{O}_{2}$ & $1 \mathrm{C}$ & 100 & 178 & 128 at $10 \mathrm{C}$ & {$[\mathrm{S} 2]$} \\
$\mathrm{Li}_{1.2} \mathrm{Mn}_{0.54} \mathrm{Ni}_{0.13} \mathrm{Co}_{0.13} \mathrm{O}_{2}$ & $1 \mathrm{C}$ & 100 & 160 & 180 at $5 \mathrm{C}$ & {$[\mathrm{S} 3]$} \\
$\mathrm{Li}_{1.2} \mathrm{Mn}_{0.6} \mathrm{Ni}_{0.2} \mathrm{O}_{2}$ & $1 \mathrm{C}$ & 100 & 210 & 140 at $5 \mathrm{C}$ & {$[\mathrm{S} 4]$} \\
$\mathrm{Li}_{1.2} \mathrm{Mn}_{0.54} \mathrm{Ni}_{0.13} \mathrm{Co}_{0.13} \mathrm{O}_{2}$ & $1 \mathrm{C}$ & 200 & 153 & 157.5 at $2 \mathrm{C}$ & {$[\mathrm{S} 5]$} \\
$\mathrm{Li}_{1.2} \mathrm{Mn}_{0.57} \mathrm{Ni}_{0.2} \mathrm{Co}_{0.12} \mathrm{O}_{2}$ & $0.3 \mathrm{C}$ & 100 & 210 & 130 at $10 \mathrm{C}$ & {$[\mathrm{S} 6]$} \\
$\mathrm{Li}_{1.2} \mathrm{Mn}_{0.6} \mathrm{Ni}_{0.2} \mathrm{O}_{2}$ & $0.1 \mathrm{C}$ & 100 & 230 & 133 at $5 \mathrm{C}$ & {$[\mathrm{S} 7]$} \\
$\mathrm{Li}_{1.2} \mathrm{Mn}_{0.54} \mathrm{Ni}_{0.16} \mathrm{Co}_{0.1} \mathrm{O}_{2}$ & $1 \mathrm{C}$ & 100 & 179 & 120 at $5 \mathrm{C}$ & {$[\mathrm{S} 8]$} \\
$\mathrm{Li}_{1.2} \mathrm{Mn}_{0.54} \mathrm{Ni}_{0.13} \mathrm{Co}_{0.13} \mathrm{O}_{2}$ & $1 \mathrm{C}$ & 150 & 230.3 & 174.6 at $5 \mathrm{C}$ & This \\
\hline
\end{tabular}


Table S5. Values of $R_{s}$ and $R_{c t}$ acquired from equivalent circuit fitting of electrochemical impedance spectra of the $\mathrm{Li} / \mathrm{LMNC}, \mathrm{Li} / \mathrm{CN}-\mathrm{LMNC}$ and $\mathrm{Li} / \mathrm{PCN}-\mathrm{LMNC}$ cells.

\begin{tabular}{ccc}
\hline Samples & $\mathrm{R}_{\mathrm{s}}(\Omega)$ & $\mathrm{R}_{\mathrm{ct}}(\Omega)$ \\
\hline LMNC & 3.6 & 92.0 \\
CN-LMNC & 2.1 & 95.6 \\
PCN-LMNC & 2.0 & 76.0 \\
\hline
\end{tabular}

\section{REFERENCES}

[S1] Wang, Z.; Lin, X.; Zhang, J.; Wang, D.; Ding, C.; Zhu, Y.; Gao, P.; Huang, X.; Wen, G. Spherical Layered Li-rich Cathode Material: Unraveling the Role of Oxygen Vacancies on Improving Lithium-Ion Conductivity. J. Power sources 2020, 462, 228171.

[S2] Jiang, C.; Zou, Z. Sheet-Like $\mathrm{Li}_{1.2} \mathrm{Mn}_{0.54} \mathrm{Ni}_{0.16} \mathrm{Co}_{0.10} \mathrm{O}_{2}$ Prepared by Glucose-Urea Bubbling and Post-Annealing Process as High-Capacity Cathode of Li-Ion Batteries. Electrochi. Acta 2018, 269, 196-203.

[S3] Zheng, F.; Ou, X.; Pan, Q.; Xiong, X.; Yang, C.; Liu, M. The effect of Composite Organic Acid (Citric Acid \& Tartaric Acid) on Microstructure and Electrochemical Properties of $\mathrm{Li}_{1.2} \mathrm{Mn}_{0.54} \mathrm{Ni}_{0.13} \mathrm{Co}_{0.13} \mathrm{O}_{2}$ Li-Rich Layered Oxides. J. Power sources 2018, $346,31-39$.

[S4] Nie, L.; Liang, C.; Chen, S.; He, Y.; Liu, W.; Zhao, H.; Gao, T.; Sun, Z.; Hu, Q.; Zhang, Y.; Yu, Y.; Liu, W. Improved Electrochemical Performance of Li-Rich Layered Oxide Cathodes Enabled by a Two-Step Heat Treatment. ACS Appl. Mater. Interfaces 2021, 13, 13281-13288. 
[S5] Yang, S.; Wang, P.; Wei, H.; Tang, L.; Zhang, X.; He, Z.; Li, Y.; Tong, H.; Zheng, J.

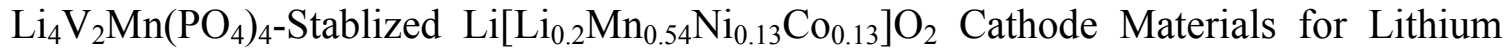
Ion Batteries. Nano Energy 2019, 63, 103889.

[S6] Erickson, E.; Sclar, H.; Schipper, F.; Liu, Jing.; Tian, R.; Ghanty, C.; Burstein, L.; Leifer, N.; Grinblat, J.; Talianker, M. Shi, J.; Lampert, J.; Markovsky, B.; Frenkel, A.; Aurbach, Doron. High-Temperature Treatment of Li-Rich Cathode Materials with Ammonia: Improved Capacity and Mean Voltage Stability during Cycling. Adv. Energy Mater. 2017, 7, 1700708 .

[S7] Zhao, S.; Sun, B.; Yan, K.; Zhang, J.; Wang, C.; Wang, G. Aegis of Lithium-Rich Cathode Materials via Heterostructured $\mathrm{LiAlF}_{4}$ Coating for High-Performance Lithium-Ion Batteries. ACS Appl. Mater. Interfaces 2018, 10, 33260-33268.

[S8] Chen, D.; Zheng, F.; Li, Liu.; Chen, M.; Zhong, X.; Li, W. Effect of $\mathrm{Li}_{3} \mathrm{PO}_{4}$ coating of layered lithium-rich oxide on electrochemical performance. J. Power sources 2017, 341, 147-155. 